\title{
Eigensolutions of the N-dimensional Schrödinger equation interacting with Varshni-Hulthén potential model
}

\author{
E. P. Inyang, E. S. William and J. A. Obu \\ Theoretical Physics Group, Department of Physics, University of Calabar, PMB 1115, Calabar, Nigeria. \\ email: williameddyphysics@gmail.com
}

Received 13 November 2020; accepted 26 November 2020

\begin{abstract}
Analytical solutions of the N-dimensional Schrödinger equation for the newly proposed Varshni-Hulthén potential are obtained within the framework of the Nikiforov-Uvarov method by using the Greene-Aldrich approximation scheme to the centrifugal barrier. The numerical energy eigenvalues and the corresponding normalized eigenfunctions are obtained in terms of Jacobi polynomials. Special cases of the potential are equally studied and their numerical energy eigenvalues are in agreement with those obtained previously with other methods. However, the behavior of the energy for the ground state and several excited states is illustrated graphically.
\end{abstract}

Keywords: N-dimensional Schrödinger equation; Nikiforov-Uvarov method; eigenvalues; eigenfunction; Varshni- Hulthén potential.

PACS: 03.65.w; 03.65.Fd; 03.65. Ge

DOI: https://doi.org/10.31349/RevMexFis.67.193

\section{Introduction}

The interactions of quantum systems with the spherically symmetric potentials have been studied by researchers since the discovery of quantum mechanics [1-3]. From the early days of quantum mechanics, the study of exactly solvable problems has attracted a considerable attention in many areas of physics, particularly in atomic physics, information theory, nuclear physics, particle physics, molecular physics and its importance cannot be overemphasized [4,5]. The exact solution of the relativistic and non-relativistic equations for most of these types of potentials is not attainable and approximations become indispensable [6]. For instance, in the case of the Schrödinger equation, when the angular momentum quantum number is present, one can resort to solve the non-relativistic equation approximately via a suitable approximation scheme [7]. Some of such approximations, yielding good results, consist of the conventional approximation scheme proposed by Greene and Aldrich [8], the improved approximation scheme by Jia et al. [9], the elegant approximation scheme [10], the Pekeris approximation [11], the improved approximation scheme by Yazarloo et al. [12], improved approximation scheme in Refs. [13-15] and in Refs. $[16,17]$.

Over the past decades, problems involving the multidimensional Schrödinger equation have been addressed by many researchers using different analytical procedures. For examples, Ntibi et al. [18] investigated the analytical solution of the D-Dimensional radial Schrödinger equation with Yukawa potential. Oyewumi et al. [19] studied the N-dimensional Pseudoharmonic oscillator. Gönül and Koçak [20] investigated explicit solutions for N-dimensional Schrödinger equations with position-dependent mass. The $\mathrm{N}$-dimensional Kratzer-Fues potential was discussed by Oyewumi [21], Ikhdair and Sever [22], studied the modi- fied Kratzer-Fues potential plus the ring shape potential in D-dimensions while Dong [23], reviewed the wave equations in higher dimensions.

The Varshni potential is greatly important with applications, cutting across nuclear physics, particle physics and molecular physics. The Varshni potential model takes the form $[24,25]$ :

$$
V_{V}=\eta_{0}-\frac{\eta_{0} \eta_{1}}{r} e^{-\delta r}
$$

where $\eta_{0}$ and $\eta_{1}$ are potential strength parameters, $\delta$ is the screening parameter and $r$ the inter-nuclear separation. The Varshni potential is a short-range repulsive potential energy.

The Hulthén potential is one of the important shortrange potentials in physics. Its relevance to diverse areas of physics including nuclear and particle physics, atomic physics, molecular physics, condensed matter and chemical physics has been of great interest and concern to researchers in recent times [26,27]. The Hulthén potential model takes the form [28]

$$
V_{H}(r)=-\frac{\eta_{2} e^{-\delta r}}{1-e^{-\delta r}}
$$

where $\delta$ is the screening parameter and $\eta_{2}$ is the potential strength constant which is sometimes identified with the atomic number when the potential is used for atomic phenomena. Many authors have obtained bound state solutions of SE with this potential. For example, Tazimi and Ghasempour [29] used the Nikiforov Uvarov (UN) method to obtain bound state solutions of the three-Dimensional Klein-Gordon equation for two model potentials. Quantization rule was employed by Ikhdair and Abu-Hasna [30] to obtain the solution to the Hulthén potential in arbitrary dimension with a new approximate scheme for the centrifugal term. Okorie et al. [31] obtain the solution of Schrödinger equation (SE) with energy- 
dependent screened Coulomb potential with the new form of Greene-Aldrich approximation using the NU method.

With the experimental proof of the Schrödinger wave equation, researchers have made great effort to solve the SE by the combination of two or more potentials, which can be used for a wider range of applications [32]. For example, Edet et al. [33] obtained bound state solutions of the SE for the modified Kratzer potential plus screened Coulomb potential including a centrifugal term. Also, Edet et al. [34] obtained any-state solutions of the SE interacting with Hellmann-Kratzer potential model. William et al. [35] obtained bound state solutions of the radial SE by the combination of Hulthén and Hellmann potential within the framework of Nikiforov-Uvarov (NU) method for any arbitrarystate, with the Greene-Aldrish approximation in the centrifugal term. Inyang et al. [36] studied any-state solutions of the SE interacting with class of Yukawa-Eckart potentials within the framework of NU method. Hence, motivated by the success of the combination of exponential-type potentials, we seek to investigate the bound state solutions of the SE by the combination of Varshni potential, of Eq. (1), and the Hulthén potential of Eq. (2) using the NU method. The resulting potential, so-called the Varshni-Hulthén potential (VHP), takes the form

$$
\begin{aligned}
V(r) & =V_{V}(r)+V_{H}(r)=\eta_{0} \\
& -\frac{\eta_{0} \eta_{1}}{r} e^{-\delta r}-\frac{\eta_{2} e^{-\delta r}}{1-e^{-\delta r}} .
\end{aligned}
$$

Considering the achievements in the previous studies, we combine the potentials to allow for more physical application and comparative analysis to existing studies of molecular physics. Also in molecular physics, it is well-known that the potential energy functions with more parameters have a tendency to fit experimental data better than those with fewer parameters [37]. The plot of the combined potential for different values of the screening parameter $\delta$ is presented in Fig. 1. However, it must be noted that the exact solution of the SE

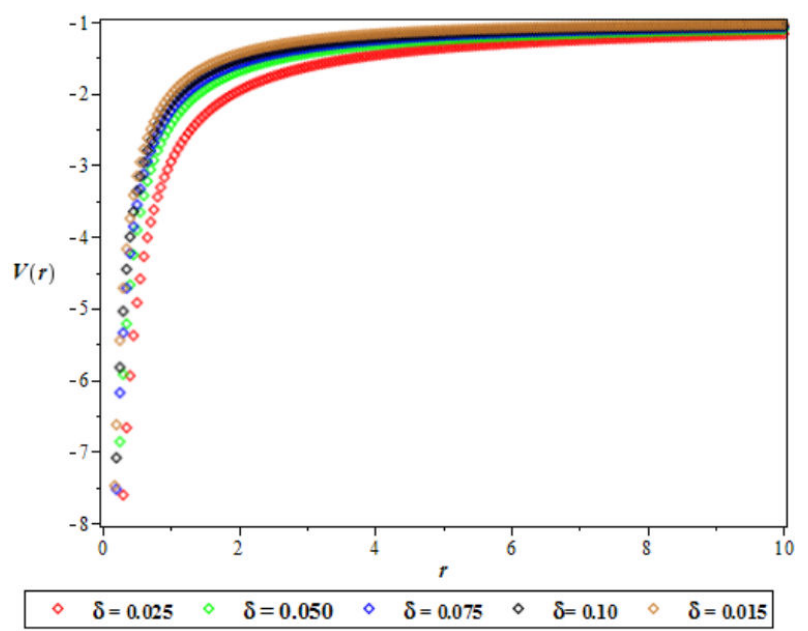

FIGURE 1. Plot of the combined potential as a function of internuclear distance for different screening parameter. We choose $\eta_{0}=\eta_{1}=-1$, and $\eta_{2}=0.025$. with the combined potentials in Eq. (3) is not possible due to the presence of the centrifugal term. Therefore, to obtain approximate solutions, we employ a suitable approximation scheme. It is found that such approximation proposed by Greene and Aldrich [8],

$$
\frac{1}{r^{2}} \approx \frac{\delta^{2}}{\left(1-e^{-\delta r}\right)^{2}}
$$

is a good approximation to the centrifugal or inverse square term for a short range potential which is valid for $\delta \square 1$.

The paper is organized as follows: In Sec. 2, we derive the bound state solutions of the SE with VHP using NU method and also derive the corresponding normalized wave function. In Sec. 3, we present the results and discussion and finally, we make a concluding remark in Sec. 4.

\section{Bound state solutions of the Schrödinger equation with Varshni plus Hulthén poten- tials}

In this study, we adopt the Nikiforov-Uvarov method which is based on solving the second-order differential equation of the hypergeometric type. The details can be found in Ref. [38]. The SE takes the form Ref. [39]

$$
\begin{aligned}
U^{/ /}{ }_{n l}(r)+ & \left(\frac{2 \mu}{\hbar^{2}}\left[E_{n l}-V(r)\right]\right. \\
& \left.-\frac{(N+2 l-1)}{4 r^{2}}\right) U_{n l}(r)=0,
\end{aligned}
$$

where $l, \mu, r, N$, and $\hbar$ and are the angular momentum, the reduced mass of the particle, inter-particle distance, the number of space, and reduced Plank constant, respectively. Substituting Eqs. (3) and (4) into Eq. (5) we obtain

$$
\begin{aligned}
& U_{n l}^{\prime \prime}(r)+\left(\frac{2 \mu}{\hbar^{2}}\left[E_{n l}-\eta_{0}+\frac{\eta_{0} \eta_{1} \delta e^{-\delta r}}{\left(1-e^{-\delta r}\right)}\right]\right. \\
& \left.-\frac{\delta^{2}(N+2 l-1)(N+3 l-3)}{4\left(1-e^{-\delta r}\right)^{2}}\right) U_{n l}(r)=0 .
\end{aligned}
$$

By using coordinate transformation

$$
x=e^{-\delta r}
$$

we obtain the differential equation as

$$
\begin{aligned}
U_{n l}^{\prime \prime}(x) & +\frac{1-x}{x(1-x)} U^{/ /}{ }_{n l}(x)+\frac{1}{x^{2}(1-x)^{2}}\left(-\left[\varepsilon+\beta_{0}\right] x^{2}\right. \\
& \left.+\left[2 \varepsilon+\beta_{0}\right] x-[\varepsilon+\gamma]\right) U(x)=0
\end{aligned}
$$


where

$$
\begin{aligned}
-\varepsilon & =\frac{2 \mu E_{n l}}{\hbar^{2} \delta^{2}}-\frac{2 \eta_{0} \mu}{\hbar^{2} \delta^{2}}, \\
\beta_{0} & =\frac{2 \eta_{0} \eta_{1} \mu}{\hbar^{2} \delta}+\frac{2 \eta_{0} \mu}{\hbar^{2} \delta^{2}}, \\
\gamma & =\frac{(N+2 l-1)(N+2 l-3)}{4} .
\end{aligned}
$$

Now that Eq. (8) and Eq. (1) of Ref. [38] are in the same shape, we have the following parameters:

$$
\begin{gathered}
\square(x)=-\left(\varepsilon+\beta_{0}\right) x^{2}+\left(2 \varepsilon+\beta_{0}\right) x-(\varepsilon+\gamma), \\
\sigma(x)=-x(1-x), \quad \tilde{\tau}(x)=1-x .
\end{gathered}
$$

Substituting Eq. (10) into Eq. (11) of Ref. [37], we obtain $\pi(x)$ as

$$
\pi(x)=-\frac{x}{2} \pm \sqrt{(A-k) x^{2}+(k+B) x+C},
$$

where

$$
A=\frac{1}{4}+\varepsilon+\beta_{0}, \quad B=-\left(2 \varepsilon+\beta_{0}\right), \quad C=\varepsilon+\gamma .
$$

To find the constant $k$, the discriminant of the expression under the square root of Eq. (11) must be equal to zero. As such we have that

$$
k=\beta_{0}-2 \gamma \pm 2 \sqrt{\varepsilon+\gamma} \sqrt{\gamma+\frac{1}{4}}
$$

Substituting Eq. (13) into Eq. (11) yields $\pi(x)$ as

$$
\pi(x)=-\frac{x}{2} \pm\left(\sqrt{\varepsilon+\gamma}+\sqrt{\gamma+\frac{1}{4}}\right) x-\sqrt{\varepsilon+\gamma}
$$

and $\tau(x)$ can be written as

$$
\begin{aligned}
\tau(x) & =1-2 x-2 \sqrt{\varepsilon+\gamma} x \\
& -2 \sqrt{\gamma+\frac{1}{4}} x+2 \sqrt{\varepsilon+\gamma}
\end{aligned}
$$

Taking the derivative of Eq. (15) with respect to $x$, we have

$$
\tau^{\prime}(x)=-2-2\left(\sqrt{\varepsilon+\gamma}+\sqrt{\gamma+\frac{1}{4}}\right) .
$$

Referring to Eq. (10) of Ref. [38], we define the constant $\gamma$ as,

$$
\begin{aligned}
& \lambda=-\frac{1}{2}-\sqrt{\varepsilon+\gamma}-\sqrt{\gamma+\frac{1}{4}}+\beta_{0} \\
& -2 \gamma-2 \sqrt{\varepsilon+\gamma} \sqrt{\gamma+\frac{1}{4}}
\end{aligned}
$$

and taking the derivative of $\sigma(x)$ with respect to $x$ from Eq. (10), we have

$$
\sigma^{\prime \prime}(x)=-2
$$

Substituting Eqs. (15) and (18) into Eq. (13) of Ref. [38], we obtain

$$
\lambda_{n}=n^{2}+n+2 n\left(\sqrt{\varepsilon+\gamma}+\sqrt{\gamma+\frac{1}{4}}\right) .
$$

By comparing Eqs. (17) and (19), using Eq. (9) yields the energy eigenvalues equation of the VHP as a function of $n$ and $l$ as

$$
E_{n l}=\eta_{0}+\frac{\delta^{2} \hbar^{2}(N+2 l-1)(N+2 l-3)}{8 \mu} \frac{\delta^{2} \hbar^{2}}{8 \mu}\left(\frac{\left.\left[n+\frac{1}{2}+\sqrt{\frac{(N+2 l-1)(N+2 l-3)}{4}+\frac{1}{4}}\right]^{2}-Q\right)^{2}}{n+\frac{1}{2}+\sqrt{\frac{(N+2 l-1)(N+2 l-3)}{4}+\frac{1}{4}}}\right.
$$

where

$$
\begin{aligned}
Q & =\left(\frac{2 \eta_{0} \eta_{1} \mu}{\hbar^{2} \delta}+\frac{2 \eta_{2} \mu}{\hbar^{2} \delta^{2}}\right) \\
& +\frac{(N+2 l-1)(N+2 l-3)}{4}
\end{aligned}
$$

To obtain the corresponding wavefunction, we consider Eq. (3) of Ref. [38], and upon substituting Eqs. (10) and (14) and integrating, we get

$$
\phi(x)=x^{\sqrt{\varepsilon+\gamma}}(1-x)^{(1 / 2)+\sqrt{(1 / 4)+\gamma}} .
$$

To get the hypergeometric function, we first determine the weight function $\rho(x)$. By substituting Eqs. (10) and (14) into Eq. (3) of Ref. [38] and after integrating, we obtain

$$
\rho(x)=x^{2 \sqrt{\varepsilon+\gamma}}(1-x)^{2 \sqrt{1 / 4+\gamma}} .
$$

Hence, by substituting Eqs. (10) and (23) into Eq. (2) yields the Rodrigues equation given as

$$
\begin{aligned}
y(x) & =N_{n l} x^{-2 \sqrt{\varepsilon+\gamma}}(1-x)^{-2 \sqrt{(1 / 4)+\gamma}} \\
& \times \frac{d^{n}}{d x^{n}}\left[x^{n+2 \sqrt{\varepsilon+\gamma}}(1-x)^{n+2 \sqrt{(1 / 4)+\gamma}}\right],
\end{aligned}
$$




\begin{tabular}{|c|c|c|c|c|}
\hline State & $\delta$ & $N=3$ & $N=4$ & $N=5$ \\
\hline \multirow[t]{5}{*}{$1 \mathrm{~s}$} & 0.025 & -1.975781250 & -1.419947195 & -1.224992578 \\
\hline & 0.050 & -1.526875000 & -1.213252345 & -1.101834379 \\
\hline & 0.075 & -1.397725694 & -1.148269303 & -1.057252171 \\
\hline & 0.100 & -1.333125000 & -1.111565340 & -1.027444000 \\
\hline & 0.150 & -1.262152778 & -1.062800124 & -0.9783441671 \\
\hline \multirow[t]{5}{*}{$2 \mathrm{~s}$} & 0.025 & -1.226250000 & -1.136131084 & -1.086889280 \\
\hline & 0.050 & -1.106875000 & -1.055772344 & -1.026860418 \\
\hline & 0.075 & -1.068611111 & -1.027534543 & -1.002633126 \\
\hline & 0.100 & -1.047656250 & -1.010587522 & -0.9858640000 \\
\hline & 0.150 & -1.023819444 & -0.9889295445 & -0.9597536576 \\
\hline \multirow[t]{5}{*}{$3 \mathrm{~s}$} & 0.025 & -1.088142361 & -1.058702155 & -1.039373438 \\
\hline & 0.050 & -1.031875000 & -1.015446221 & -1.003900782 \\
\hline & 0.075 & -1.013913966 & -1.001158640 & 0.9908992733 \\
\hline & 0.100 & -1.005902778 & -0.9950120013 & -.09844360000 \\
\hline & 0.150 & -1.004683642 & -0.9961284666 & -0.9827782293 \\
\hline \multirow[t]{5}{*}{$3 p$} & 0.025 & -1.086889280 & -1.056823915 & -1.036870313 \\
\hline & 0.050 & -1.026860418 & -1.007932642 & -0.9938898525 \\
\hline & 0.075 & -1.002633126 & -0.9842622719 & -0.9683923310 \\
\hline & 0.100 & -0.9858640000 & -0.9650092156 & -0.9444802500 \\
\hline & 0.150 & -0.9597536576 & -0.9289088370 & -0.8933044371 \\
\hline \multirow[t]{5}{*}{$3 d$} & 0.025 & -1.084383117 & -1.053693514 & -1.033115626 \\
\hline & 0.050 & -1.016831266 & -0.9954100212 & -0.9788734727 \\
\hline & 0.075 & -0.9800715659 & -0.9561018046 & -0.9346320845 \\
\hline & 0.100 & -0.9457871111 & -0.9150053890 & -0.8845475625 \\
\hline & 0.150 & -0.8699012826 & -0.8168854194 & -0.7591039275 \\
\hline \multirow[t]{5}{*}{$4 \mathrm{~s}$} & 0.025 & -1.040625000 & -0.8168854194 & -1.018280391 \\
\hline & 0.050 & -1.008906250 & -1.002307899 & -0.9968737506 \\
\hline & 0.075 & -1.002152778 & -0.9980821361 & -0.9935681755 \\
\hline & 0.100 & -1.004414062 & -1.002429482 & -0.9981750400 \\
\hline & 0.150 & -1.027517361 & -1.030202097 & -1.025835317 \\
\hline \multirow[t]{5}{*}{$4 p$} & 0.025 & -1.039373438 & -1.025825868 & -1.015778672 \\
\hline & 0.050 & -1.003900782 & -0.9948033881 & -0.9868712556 \\
\hline & 0.075 & -0.9908992733 & -0.9812129600 & -0.9710865137 \\
\hline & 0.100 & -0.9844360000 & -0.9724870563 & -0.9582753600 \\
\hline & 0.150 & -0.9827782293 & -0.9631717038 & -0.9365364617 \\
\hline
\end{tabular}

where $N_{n l}$ is the normalization constant. Equation (24) is tion is given as equivalent to

$$
P_{n}^{(2 \sqrt{\varepsilon+\gamma}, 2 \sqrt{(1 / 4)+\gamma})}(1-2 x),
$$

where $P_{n}^{\alpha, \beta}$ is the Jacobi Polynomials. Hence, the wave func-

$$
\begin{aligned}
\psi_{n l}(x) & =N_{n l} x^{\sqrt{\varepsilon+\gamma}}(1-x)^{1 / 2+\sqrt{(1 / 4)+\gamma}} \\
& \times P_{n}^{(2 \sqrt{\varepsilon+\gamma}, 2 \sqrt{(1 / 4)+\gamma})}(1-2 x) .
\end{aligned}
$$




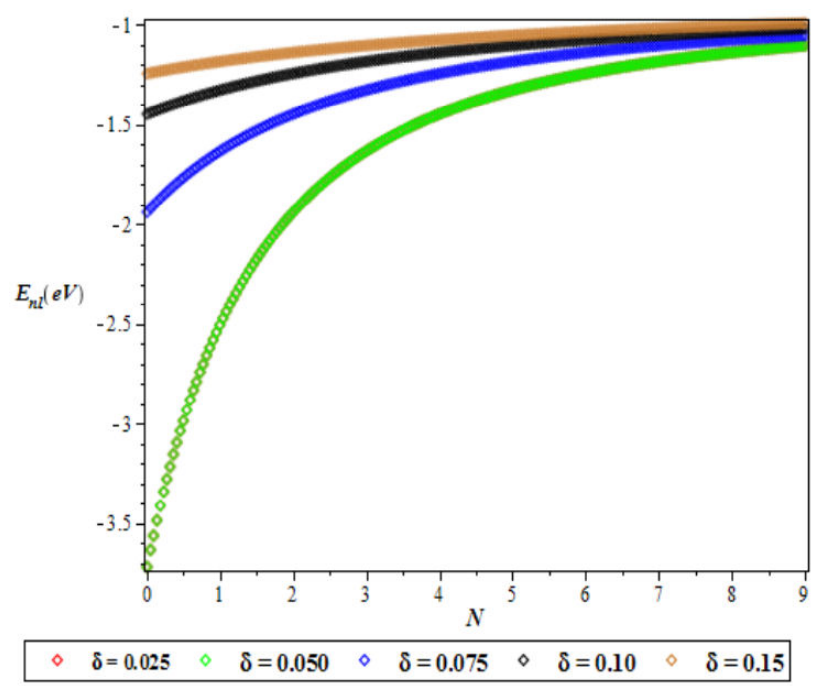

FIGURE 2. Variation of energy eigenvalues as function of $\mathrm{N}$ for different $l$ and $\delta$. We choose $a=b=-1$, and $c=0.025$

Using the normalization condition, we obtain the normalization constant as follows:

$$
\int_{0}^{\infty}\left|\psi_{n l}(r)\right|^{2} d r=1 .
$$

From our coordinate transformation of Eq. (7), we have that

$$
-\frac{1}{\delta x} \int_{1}^{0}\left|\psi_{n l}\right|^{2} d x=1 .
$$

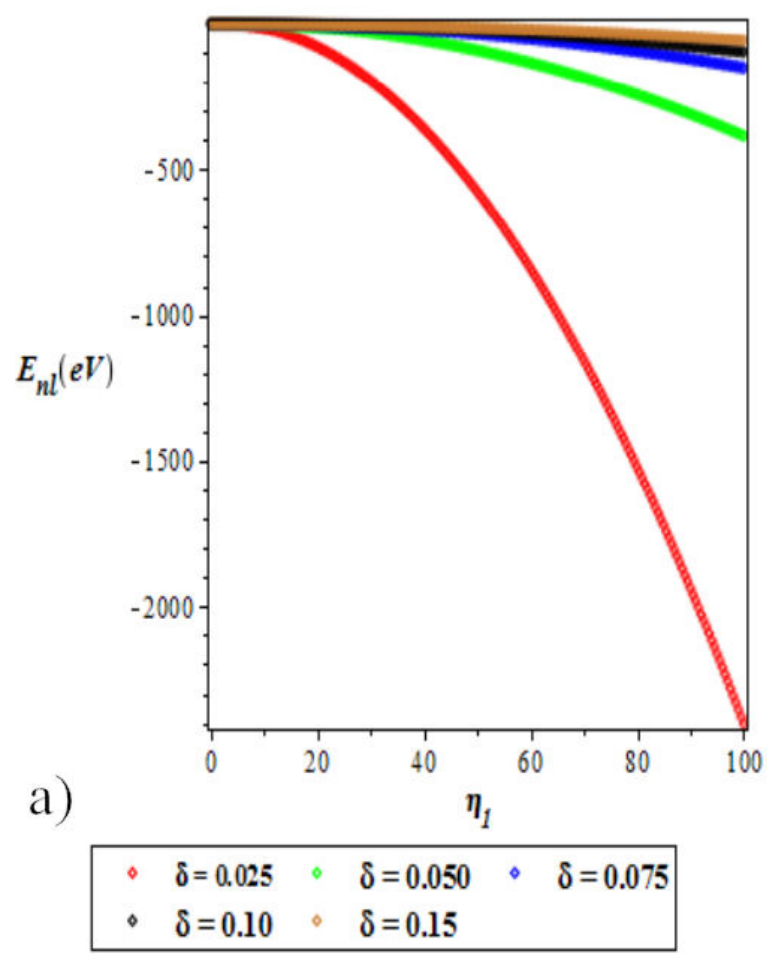

By letting, $y=1-2 x$, we have

$$
\begin{gathered}
\frac{N_{n l}^{2}}{\delta} \int_{-1}^{1}\left(\frac{1-y}{2}\right)^{2 \sqrt{\varepsilon+\gamma}}\left(\frac{1+y}{2}\right)^{1+2 \sqrt{(1 / 4)+\gamma}} \\
\quad \times\left(P_{n}^{(2 \sqrt{\varepsilon+\gamma}, 2 \sqrt{(1 / 4)+\gamma} y)}\right)^{2} d y=1
\end{gathered}
$$

Let

$$
\begin{gathered}
v=1+2 \sqrt{\frac{1}{4}+\gamma}, \quad \text { and } \\
\mu-1=2 \sqrt{\frac{1}{4}+\gamma}, \quad u=2 \sqrt{\varepsilon+\gamma}
\end{gathered}
$$
have

By substituting Eq. (29) into Eq. (28), using Eq. (30), we

$$
\begin{aligned}
& \frac{N_{n l}^{2}}{\delta} \int_{-1}^{1}\left(\frac{1-y}{2}\right)^{u}\left(\frac{1+y}{2}\right)^{v} \\
& \quad \times\left(P_{n}^{(2 u, v-1)} y\right)^{2} d y=1 .
\end{aligned}
$$

According to Onate and Ojonubah [40], integral of the form in Eq. (31) can be expressed as

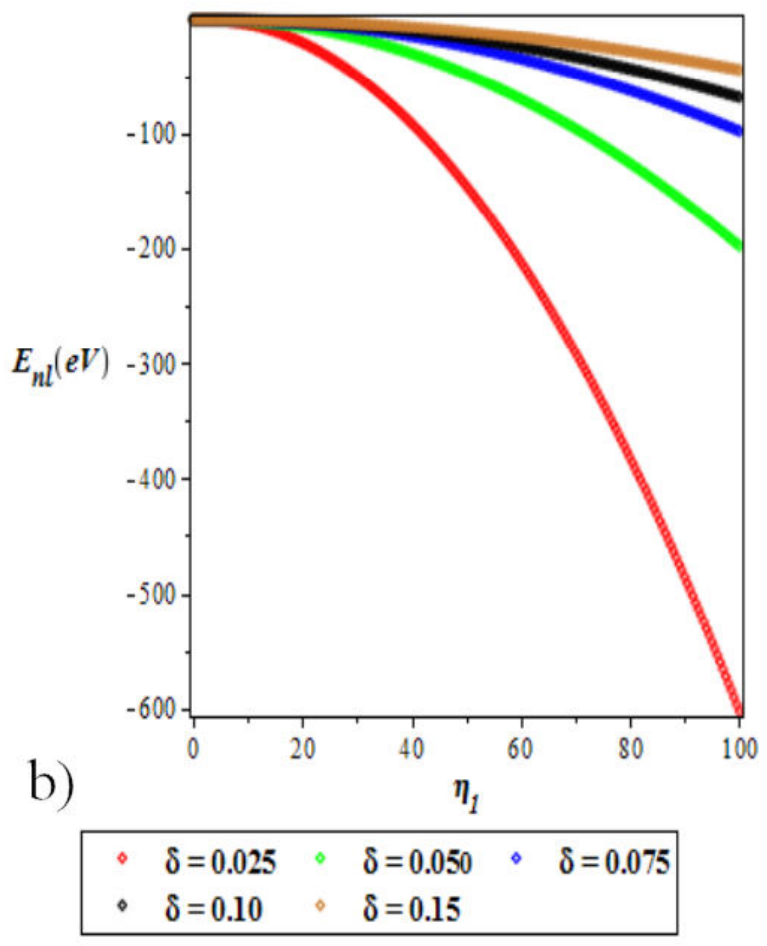

FIGURE 3. a) The plot of the ground state energy spectra for various $l$ as a function of $\eta_{0}$. b) Variation of the first excited state energy spectra for different $l$ as a function of $\eta_{0}$. We choose $a=b=-1, c=0.025$ and $\delta=0.025$ for the ground and excited states. 
a)

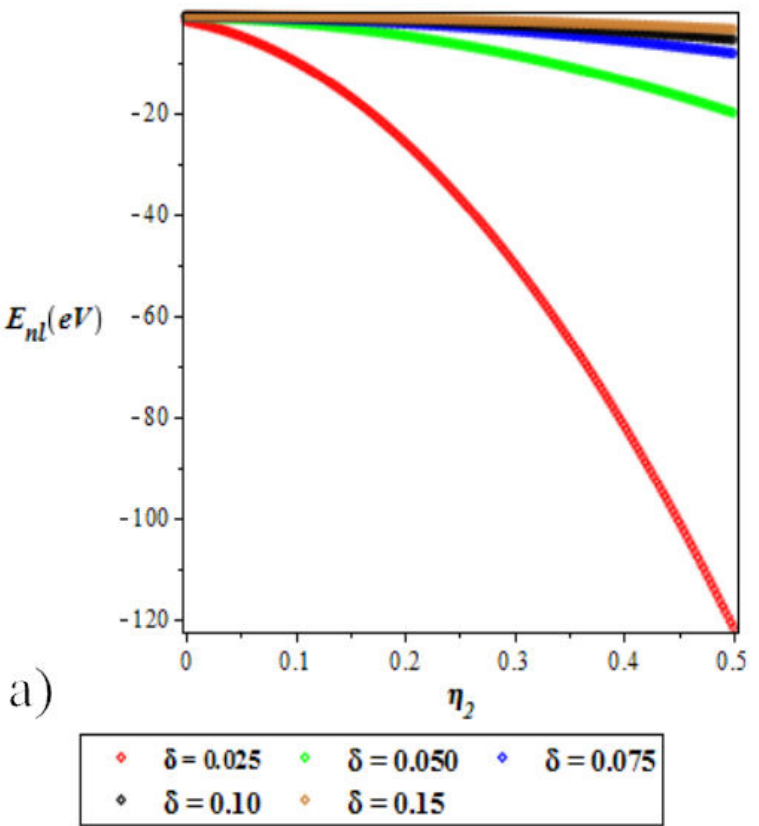

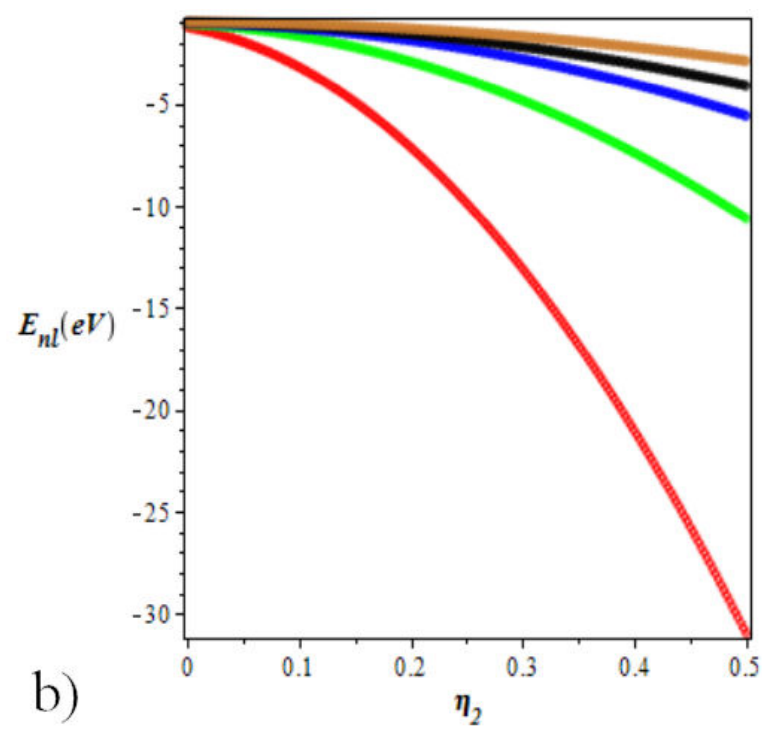

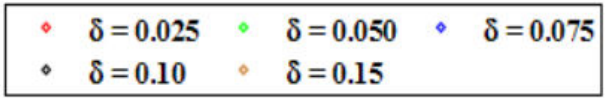

FIGURE 4. a) Variation of the ground state energy spectra for various $l$ as a function of $\eta_{1}$. b) A plot of the first excited state energy spectra for various $l$ as a function of $\eta_{1}$. We choose $a=b=-1, c=0.025$ and $\delta=0.025$ for the ground and excited states.
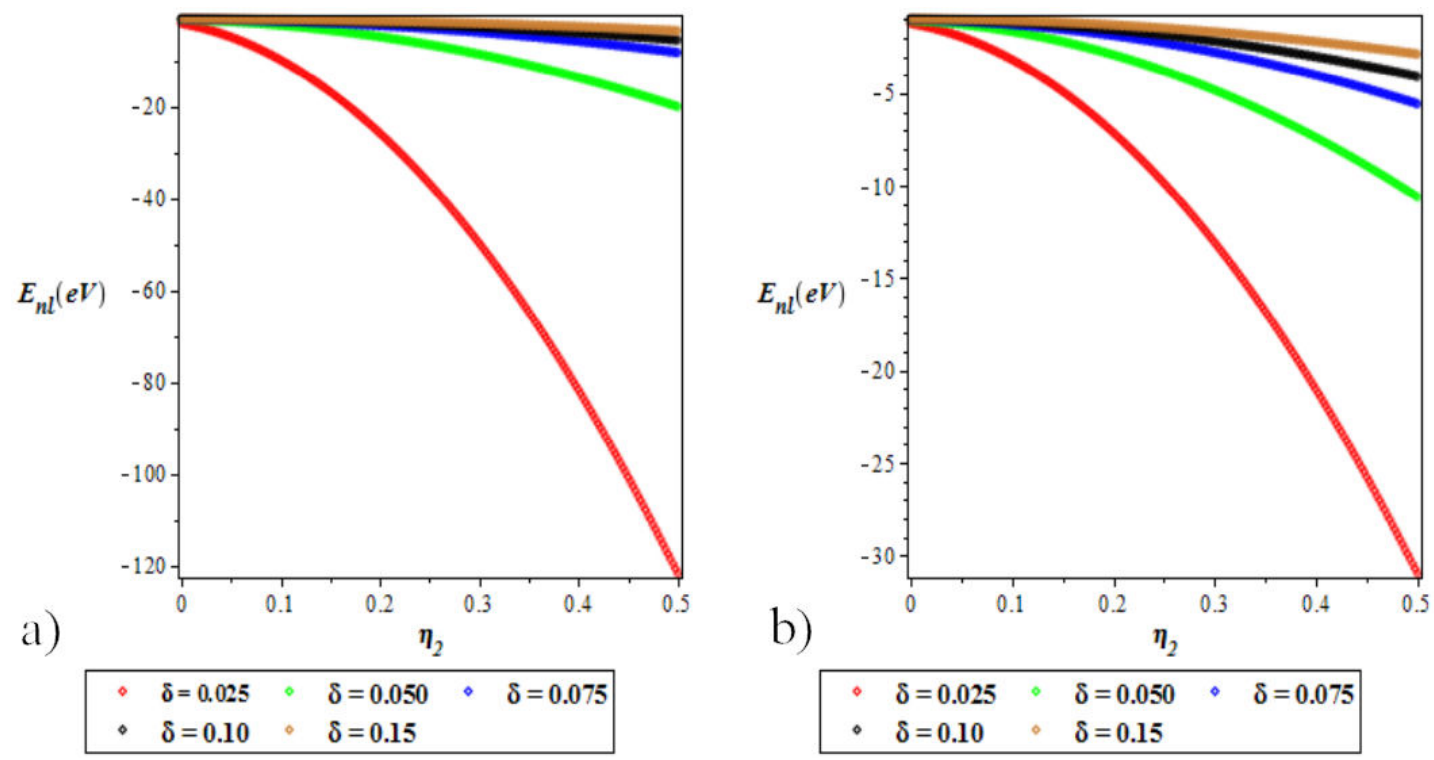

FIGURE 5. a) Variation of the ground state energy spectra for various $l$ as a function of $\eta_{2}$. b) The plot of the first excited state energy spectra for various $l$ as a function of $\eta_{2}$. We choose $a=b=-1, c=0.025$ and $\delta=0.025$ for the ground and excited states.

$$
\begin{aligned}
& \int_{-1}^{1}\left(\frac{1-p}{2}\right)^{x}\left(\frac{1+p}{2}\right)^{y}\left(P_{n}^{(2 x, y-1)} p\right)^{2} d p \\
& =\frac{2 \Gamma(x+n+1) \Gamma(y+n+1)}{n ! x \Gamma(x+y+n+1)}
\end{aligned}
$$

Hence, by comparing Eq. (31) with the standard integral of Eq. (32), we obtain the normalization constant as

$$
N_{n l}=\sqrt{\frac{n ! u \delta \Gamma(u+v+n+1)}{2 \Gamma(u+n+1) \Gamma(v+n+1)}} .
$$

\section{Discussion}

Solution of the radial N-dimensional SE for the newly proposed potential obtained by the superposition of Varshni and 
TABLE II. Energy eigenvalues (eV) of the Varshni-Hulthen potential for $N=3$ with $\hbar=2 \mu=1$.

\begin{tabular}{|c|c|c|c|c|}
\hline State & $\delta$ & $\eta_{0}=-1, \eta_{1}=-2, \eta_{2}=0.050$ & $\eta_{0}=-2, \eta_{1}=-1, \eta_{2}=0.075$ & $\eta_{0}=\eta_{1}=-2, \eta_{2}=0.10$ \\
\hline \multirow[t]{5}{*}{$1 \mathrm{~s}$} & 0.025 & -4.951406250 & -8.189531250 & -17.90265625 \\
\hline & 0.050 & -3.178125000 & -4.979375000 & -10.85562500 \\
\hline & 0.075 & -2.682934026 & -4.144531249 & -8.920017362 \\
\hline & 0.100 & -2.445000000 & -3.763125000 & -8.012500000 \\
\hline & 0.150 & -2.199236110 & -3.391874999 & -7.115069440 \\
\hline \multirow[t]{5}{*}{$2 \mathrm{~s}$} & 0.025 & -1.951875000 & -3.502500000 & -5.903125000 \\
\hline & 0.050 & -1.492500000 & -2.684375000 & -4.107500000 \\
\hline & 0.075 & -1.353819444 & -2.461250000 & -3.590902778 \\
\hline & 0.100 & -1.280625000 & -2.352656250 & -3.332500000 \\
\hline & 0.150 & -1.195277778 & -2.236875000 & -3.048611110 \\
\hline \multirow[t]{5}{*}{$2 p$} & 0.025 & -1.950609766 & -3.501230860 & -5.901844140 \\
\hline & 0.050 & -1.487412504 & -2.679271879 & -4.102318752 \\
\hline & 0.075 & -1.342319880 & -2.449715279 & -3.579121962 \\
\hline & 0.100 & -1.260100250 & -2.332069000 & -3.311350250 \\
\hline & 0.150 & -1.148818125 & -2.190274722 & -3.000182708 \\
\hline \multirow[t]{5}{*}{$3 \mathrm{~s}$} & 0.025 & -1.397100694 & -2.635225694 & -3.681684028 \\
\hline & 0.050 & -1.183125000 & -2.262152778 & -2.860625000 \\
\hline & 0.075 & -1.113937114 & -2.155781250 & -2.610279707 \\
\hline & 0.100 & -1.076111111 & -2.102569444 & -2.476944444 \\
\hline & 0.150 & -1.034359568 & -2.047986111 & -2.320563272 \\
\hline \multirow[t]{5}{*}{$3 p$} & 0.025 & -1.395844140 & -2.633967404 & -3.680420530 \\
\hline & 0.050 & -1.178089585 & -2.257110418 & -2.855547918 \\
\hline & 0.075 & -1.102593775 & -2.144422285 & -2.598811367 \\
\hline & 0.100 & -1.055933444 & -2.082364000 & -2.456489000 \\
\hline & 0.150 & -0.9889920835 & -2.002556127 & -2.274320787 \\
\hline \multirow[t]{5}{*}{$3 d$} & 0.025 & -1.393331033 & -2.631450825 & -3.677893532 \\
\hline & 0.050 & -1.168018766 & -2.247025710 & -2.845393765 \\
\hline & 0.075 & -1.079907212 & -2.121704475 & -2.575874807 \\
\hline & 0.100 & -1.015578778 & -2.041953778 & -2.415578778 \\
\hline & 0.150 & -0.8982647085 & -1.911703752 & -2.181843412 \\
\hline \multirow[t]{5}{*}{$4 \mathrm{~s}$} & 0.025 & -1.203750000 & -2.332500000 & -2.905000000 \\
\hline & 0.050 & -1.078125000 & -2.117656250 & -2.427500000 \\
\hline & 0.075 & -1.037361111 & -2.056250000 & -2.274444444 \\
\hline & 0.100 & -1.017656250 & -2.028164062 & -2.190625000 \\
\hline & 0.150 & -1.007569444 & -2.011406250 & -2.095277778 \\
\hline \multirow[t]{5}{*}{$4 p$} & 0.025 & -1.202496484 & -2.331245508 & -2.903742578 \\
\hline & 0.050 & -1.073107813 & -2.112635157 & -2.422459376 \\
\hline & 0.075 & -1.026072450 & -2.044952550 & -2.263085471 \\
\hline & 0.100 & -0.9976000625 & -2.008092250 & -2.170412562 \\
\hline & 0.150 & -0.9625842189 & -1.966385868 & -2.049800365 \\
\hline
\end{tabular}


TABLE III. Energy eigenvalues (eV) of Varshni potential as a function of the screening parameter $(\delta)$ for in atomic mass units $(\hbar=\mu=1)$.

\begin{tabular}{|c|c|c|c|c|c|}
\hline State & $\delta$ & $\begin{array}{l}\text { Present method } \\
\qquad a=b=-1\end{array}$ & $\begin{array}{c}\text { Present method } \\
a=-1, b=-2\end{array}$ & $\begin{array}{l}\text { Present method } \\
a=-2, b=-1\end{array}$ & $\begin{array}{c}\text { (FM) [40] } \\
a=b=-1\end{array}$ \\
\hline \multirow[t]{3}{*}{$1 \mathrm{~s}$} & 0.001 & -1.063124562 & -2.001000250 & -3.001000250 & \\
\hline & 0.050 & -1.092656250 & -2.050625000 & -3.050625000 & \\
\hline & 0.100 & -1.120625000 & -2.102500000 & -3.102500000 & \\
\hline \multirow[t]{3}{*}{$2 \mathrm{~s}$} & 0.001 & -1.063001000 & -1.251001000 & -2.251001000 & \\
\hline & 0.050 & -1.090000000 & -1.302500000 & -2.302500000 & \\
\hline & 0.100 & -1.122500000 & -1.360000000 & -2.360000000 & \\
\hline \multirow[t]{3}{*}{$2 p$} & 0.001 & -1.063124562 & -1.251249562 & -2.251249562 & -1.0617502 \\
\hline & 0.050 & -1.092656250 & -1.311406250 & -2.311406250 & -1.0617502 \\
\hline & 0.100 & -1.120625000 & -1.370625000 & -2.370625000 & -0.9900000 \\
\hline \multirow[t]{3}{*}{$3 \mathrm{~s}$} & 0.001 & -1.028280028 & -1.112113361 & -2.112113361 & \\
\hline & 0.050 & -1.058402778 & -1.166736111 & -2.166736111 & \\
\hline & 0.100 & -1.100277778 & -1.233611111 & -2.233611111 & \\
\hline \multirow[t]{3}{*}{$3 p$} & 0.001 & -1.028334111 & -1.112223000 & -2.112223000 & \\
\hline & 0.050 & -1.057500000 & -1.168611111 & -2.168611111 & \\
\hline & 0.100 & -1.091111111 & -1.230000000 & -2.230000000 & - \\
\hline \multirow[t]{3}{*}{$3 d$} & 0.001 & -1.028386250 & -1.112330694 & -2.112330694 & -1.0269447 \\
\hline & 0.050 & -1.051736111 & -1.165625000 & -2.165625000 & -0.9867361 \\
\hline & 0.100 & -1.062500000 & -1.206944444 & -2.206944444 & -0.9469444 \\
\hline \multirow[t]{3}{*}{$4 \mathrm{~s}$} & 0.001 & -1.016129000 & -1.063504000 & -2.063504000 & - \\
\hline & 0.050 & -1.050625000 & -1.122500000 & -2.122500000 & - \\
\hline & 0.100 & -1.105625000 & -12025000000 & -2.202500000 & - \\
\hline \multirow[t]{3}{*}{$4 p$} & 0.001 & -1.016158766 & -1.063565016 & -2.063565016 & -1.0150656 \\
\hline & 0.050 & -1.048476562 & -1.121914062 & -2.121914062 & -09951563 \\
\hline & 0.100 & -1.093906250 & -1.193906250 & -2.193906250 & -0.990000 \\
\hline \multirow[t]{3}{*}{$4 d$} & 0.001 & -1.028386250 & -1.063624062 & -2.063624063 & -1.0149391 \\
\hline & 0.050 & -1.051736111 & -1.116406250 & -2.116406250 & -0.99851563 \\
\hline & 0.100 & -1.062500000 & -1.063624062 & -2.165625000 & -0.9625000 \\
\hline \multirow[t]{3}{*}{$4 f$} & 0.001 & -1.016212391 & -1.063681141 & -2.063681141 & -1.0147502 \\
\hline & 0.050 & -1.029414062 & -1.1059765662 & -2.1059765662 & -0.9725000 \\
\hline & 0.100 & -1.011406250 & -1.117656250 & -2.117656250 & -0.9306250 \\
\hline
\end{tabular}

4, and 5, respectively. We observed that the energy increases as the screening parameters and $N$ increases. In Table II, we have presented the energy eigenvalues $(\mathrm{eV})$ with $\hbar=2 \mu=1$ of the VHP for $N=3$ with three different values of the potential range: $\eta_{0}=-1 ; \eta_{1}=-1, \eta_{2}=0.050: \eta_{0}=-2$, $\eta_{1}=-1, \eta_{2}=0.075$ and $\eta_{0}=\eta_{1}=-2, \eta_{2}=0.10$, respectively, and observed that the energy increases as the screening parameters increases for constant $N$. We have plotted the graph of energy eigenvalues against the number of dimensional space, potential strength, screening parameter, and the reduced mass in the ground and excited states. In Fig. 2, we show the variation of energy as a function of and ob- served that the energy increases as both and increases. From Figs. 3a) and b) - Figs. 5a) and b) respectively, we plotted the ground and excited states energy eigenvalues of the different quantum states as a function of the VHP strengths. We observed that there is a decrease in energy in both the ground and excited states as the potential strength, $\eta_{0}, \eta_{1}$, and $\eta_{2}$ respectively, increases.

The variation of energy eigenvalues of VHP as a function of the screening parameter is as shown in Fig. 6a) and 6b). We observed that the energy increases as the screening parameter increases in the ground and excited states. In Fig. 7a) and $7 \mathrm{~b}$ ), we show the variation of energy eigenvalues as a 


\begin{tabular}{cccccc}
\hline \hline TABLE IV. Energy eigenvalues $(\mathrm{eV})$ of the Varshni-Hulthen potential for $N=3$ with $\hbar=2 \mu=1$. & \\
\hline State & $\delta$ & Present method & EQR [42] & AIM[43] & Numerical [44] \\
\hline $2 \mathrm{p}$ & 0.025 & -0.1127611 & -0.1128125 & -0.1128125 & -0.112760 \\
& 0.050 & -0.1010442 & -0.1012500 & -0.1012500 & -0.101042 \\
& 0.075 & -0.0898495 & -0.0903125 & -0.0903125 & -0.089847 \\
& 0.100 & -0.0791769 & -0.0800000 & -0.0800000 & -0.079179 \\
& 0.150 & -0.0593981 & -0.0612500 & -0.0612500 & -0.059441 \\
\hline $3 p$ & 0.025 & -0.0437072 & -0.0437590 & -0.0437590 & -0.043706 \\
& 0.050 & -0.0331623 & -0.0333681 & -0.033368 & -0.033164 \\
& 0.075 & -0.0239207 & -0.0243837 & -0.0243837 & -0.023939 \\
& 0.100 & -0.0159825 & -0.0168056 & -0.0168056 & -0.016053 \\
& 0.150 & -0.0040162 & -0.0058681 & -0.0058681 & -0.004466 \\
\hline $3 \mathrm{~d}$ & 0.025 & -0.0436044 & -0.0437587 & -0.0437587 & -0.043603 \\
& 0.050 & -0.0327508 & -0.0333681 & -0.0333681 & -0.032753 \\
& 0.075 & -0.0229948 & -0.0243837 & -0.0243837 & -0.023030 \\
& 0.100 & -0.0143364 & -0.0168055 & -0.0168055 & -0.014484 \\
& 0.150 & -0.0003124 & -0.0058681 & -0.0058681 & -0.001396 \\
\hline $4 \mathrm{p}$ & 0.025 & -0.0199486 & -0.0200000 & -0.0200000 & -0.019948 \\
& 0.050 & -0.0110442 & -0.0112500 & -0.0112500 & -0.011058 \\
& 0.075 & -0.0045370 & -0.0050000 & -0.0050000 & -0.004621 \\
& 0.100 & -0.0004269 & -0.0012500 & -0.0012500 & -0.000755 \\
\hline $4 \mathrm{f}$ & 0.025 & -0.0198457 & -0.0200000 & -0.0200000 & -0.019846 \\
& 0.050 & -0.0106327 & -0.0112500 & -0.0112500 & -0.010667 \\
& 0.075 & -0.0036111 & -0.0050000 & -0.0050000 & -0.003834 \\
\hline 0.025 & -0.0196914 & -0.0200000 & -0.0200000 & -0.019691 \\
& 0.050 & -0.0100154 & -0.0112500 & -0.0112500 & -0.010062 \\
& 0.075 & -0.0022222 & -0.0050000 & -0.0050000 & -0.002556 \\
\hline
\end{tabular}

as a function of the reduced mass. It is observed that there is a decrease in the ground and excited states energy for different quantum states as the reduced mass, $\mu$ increases.

\section{Special cases}

1. When we set $\eta_{2}=0$ in Eq. (20), we obtain the energy eigenvalues for the Varshni potential,

$$
E_{n l}=\eta_{0}+\frac{\delta^{2} \hbar^{2}(N+2 l-3)}{8 \mu}-\frac{\delta^{2} \hbar^{2}}{8 \mu}\left(\frac{\left(n+\frac{1}{2}+\sqrt{\frac{(N+2 l-1)(N+2 l-3)}{4}+\frac{1}{4}}\right)^{2}-P}{n+\frac{1}{2}+\sqrt{\frac{(N+2 l-1)(N+2 l-3)}{4}+\frac{1}{4}}}\right)^{2}
$$

where

$$
P=\frac{2 \eta_{0} \eta_{1} \mu}{\hbar^{2} \delta}+\frac{(N+2 l-1)(N+2 l-3)}{4} .
$$

The numerical energy eigenvalues of Eqs. (34) and (35) for as presented in Table III, were computed for three different potential strengths; for $1 \mathrm{~s}, 2 \mathrm{~s}, 2 \mathrm{p}, 3 \mathrm{~s}, 3 \mathrm{p}, 3 \mathrm{~d}, 4 \mathrm{~s}, 4 \mathrm{p}, 4 \mathrm{~d}$ and $4 \mathrm{f}$ states and were found to be in conformity when compared with the work of Ebonwonyi et al. [41] using formula method.

2. If we set $\eta_{0}=\eta_{1}=0$ in Eq. (20), we obtain the energy eigenvalue equation for Hulthén potential 


$$
E_{n l}=\frac{\delta^{2} \hbar^{2}(N+2 l-1)(N+2 l-3)}{8 \mu}-\frac{\delta^{2} \hbar^{2}}{8 \mu}\left(\frac{\left(n+\frac{1}{2}+\sqrt{\frac{(N+2 l-1)(N+2 l-3)}{4}+\frac{1}{4}}\right)^{2}-R}{n+\frac{1}{2}+\sqrt{\frac{(N+2 l-1)(N+2 l-3)}{4}+\frac{1}{4}}}\right)^{2}
$$

where

$$
R=\frac{2 \eta_{2} \mu}{\hbar^{2} \delta^{2}}+\frac{(N+2 l-1)(N+2 l-3)}{4} .
$$

The numerical energy eigenvalues of Hulthén potential presented in Eqs. (36) and (37) for $N=3$ were computed for $2 \mathrm{p}, 3 \mathrm{p}, 3 \mathrm{~d}, 4 \mathrm{p}, 4 \mathrm{~d}$ and $4 \mathrm{f}$ states and was compare with the work of Qiang et al. [42] using EQR, Bayrak et al. [43] using AIM, and Ikhdair [44] using numerical methods and were found to be in excellent agreement, as presented in Table IV.

3. If we set $N=l=0$ in Eq. (20), we obtain the energy eigenvalue equation for the s-wave of VHP as

$$
E_{n l}=\eta_{0}-\frac{\delta^{2} \hbar^{2}}{8 \mu}\left(\frac{\left[n+\frac{1}{2}\right]^{2}-\left[\frac{2 \eta_{0} \eta_{1} \mu}{\hbar^{2} \delta}+\frac{2 \eta_{2} \mu}{\hbar^{2} \delta^{2}}\right]}{n+\frac{1}{2}}\right)^{2}
$$

\section{Conclusion}

The analytical solutions of the $\mathrm{N}$-dimensional Schrödinger equation for the newly proposed Varshni-Hulthén potential are obtained via NU method by using Greene-Aldrich approximation scheme to the centrifugal barrier. The numerical energy eigenvalues and the corresponding normalized eigenfunctions are obtained for various values of orbital angular momentum quantum number and space dimension. We have also obtained the numerical energy eigenvalues for two special cases of the newly proposed potential and their results were found to be in agreement with the existing literature. However, the behavior of the energy for the ground state and several excited states is illustrated graphically. Therefore, studying the analytical solution of the N-dimensional Schrödinger equation for the newly proposed Varshni-Hulthen potential could provide valuable information on the quantum mechanics dynamics at atomic and molecular physics and opens new window.

1. A. Özfidan, Approximate Bound State Solutions of the Hellmann Plus Kratzer Potential in N-dimensional space, Gazi Univ. J. Sci. 33 (2020) 791, https://doi.org/10. 35378 /gujs.672684

2. W. A. Yahya and K. J. Oyewumi, Thermodynamic properties and approximate solutions of the $\ell$-state Pöschl-Tellertype potential, J. Asoc. Arab Univ. Basic Appl. Sci. 21 (2016) 53, https://doi.org/10.1016/j.jaubas. 2015.04 .001

3. K. J. Oyewumi and O. J. Oluwadare, The scattering phase shifts of the Hulthén-type potential plus Yukawa potential, Eur. Phys. J. Plus 131 (2016) 295, https://doi.org/10.1140/ epjp/i2016-16295-y

4. A. N. Ikot et al., Bound state solutions of the Schrödinger equation with energydependent molecular Kratzer potential via asymptotic iteration method, Eclét. Quím. J. 45 (2020) 65, https://doi.org/10.26850/1678-4618eqj. v45.1.2020.p65-77

5. H. Louis et al., Solutions to the Dirac Equation for ManningRosen Plus Shifted Deng- Fan Potential and Coulomb-Like Tensor Interaction Using Nikiforov-Uvarov Method, Int. J. Chem. 10 (2018) 99, https: / /doi.org/10.5539/ijc. v10n3p99

6. H. Louis, B. I. Ita, and N. I Nzeata, Approximate solution of the Schrödinger equation with Manning-Rosen plus Hellmann potential and its thermodynamic properties using the proper quantization rule, Eur. Phys. J. Plus 134 (2019) 315, https : //doi.org/10.1140/epjp/i2019-12835-3

7. J. Lu, Analytic Quantum Mechanics of Diatomic Molecules with Empirical Potentials, Phys. Scr. 72 (2005) 349, https://doi.org/10.1238/Physica.Regular. $072 a 00349$

8. R. L. Greene and C. Aldrich, Variational wave functions for a screened Coulomb potential, Phys. Rev. A 14 (1976) 2363, https://doi.org/10.1103/PhysRevA.14.2363

9. C.-S. Jia, T. Chen, and L.-G. Cui, Approximate analytical solutions of the Dirac equation with the generalized PöschlTeller potential including the pseudo-centrifugal term, Phys. Lett. A 373 (2009) 1621, https://doi.org/10.1016/ j.physleta.2009.03.006

10. E. L. Hill, The Theory of Vector Spherical Harmonics, Am. J. Phys. 22 (1954) 211, https://doi.org/10.1119/1. 1933682

11. C. L. Pekeris, The Rotation-Vibration Coupling in Diatomic Molecules, Phys. Rev. 45 (1934) 98, https://doi.org/ 10.1103/PhysRev.45.98 
12. B. H. Yazarloo, H. Hassanabadi, and S. Zarrinkamar, Oscillator strengths based on the Möbius square potential under Schrödinger equation, Eur. Phys. J. Plus 127 (2012) 51, https://doi.org/10.1140/epjp/ i2012-12051-9

13. W.-C. Qiang and S.-H. Dong, Analytical approximations to the solutions of the Manning-Rosen potential with centrifugal term, Phys. Lett. A 368 (2007) 13, https://doi.org/10. $1016 / j \cdot$ physleta.2007.03.057

14. S.-H. Dong, W.-C. Qiang, G.-H. Sun, and V. B. Bezerra, Analytical approximations to the $l$-wave solutions of the Schrödinger equation with the Eckart potential, $J$. Phs. A 40 (2007) 10535, https://doi.org/10.1088/ $1751-8113 / 40 / 34 / 010$

15. G.-F. Wei, C.-Y. Long, and S.-H. Dong, The scattering of the Manning-Rosen potential with centrifugal term, Phys. Lett. A 372 (2008) 2592, https://doi.org/10.1016/ j.physleta.2007.12.042.

16. S.-H. Dong and X.-Y. Gu, Arbitrary $l$ state solutions of the Schrödinger equation with the Deng-Fan molecular potential, J. Phys.: Conf. Ser. 96 (2008) 012109, https : / / doi.org/ $10.1088 / 1742-6596 / 96 / 1 / 012109$

17. S.-H. Dong, W.-C. Qiang, and J. García-Ravelo, Analytical approximations to the Schrödinger equation for a second Pöschl-Teller-like potential with centrifugal term, Int. J. Mod. A 23 (2008) 1537, https://doi.org/10.1142/ S0217751X0803944X

18. J. E. Ntibi, E. P. Inyang, E. P. Inyang, and E. S. William, Relativistic treatment of Ddimensional Klein-Gordon equation with Yukawa potential, Int. J. Innov. Sci. Eng. Technol. 7 (2020) 28.

19. K. J. Oyewumi, F. O. Akinpelu, and A. D. Agboola, Exactly Complete Solutions of the Pseudoharmonic Potential in $N$ Dimensions, Int. J. Theor. Phys. 47 (2008) 1039, https: //doi.org/10.1007/s10773-007-9532-x

20. B. Gönül and M. Koçak, Explicit solutions for $N$-dimensional Schrödinger equations with position-dependent mass, J. Math. Phys. 47 (2006) 102101, https://doi.org/10.1063/ 1.2354333 .

21. K. J. Oyewumi, Analytical Solutions of the Kratzer-Fues Potential in an Arbitrary Number of Dimensions, Found. Phys. Lett. 18 (2005) 75, https://doi.org/10.1007/ s10702-005-2481-9.

22. S. M. Ikhdair and R. Sever, Exact bound states of the Ddimensional Klein-Gordon equation with equal scalar and vector ring-shaped pseudoharmonic potential, Int. J. Mod. Phys. C 19 (2008) 1425, https://doi.org/10.1142/ S0129183108012923

23. S.-H. Dong, Wave Equations in Higher Dimensions (Springer, Dordrecht, 2011), https://doi.org/10.1007/ 978-94-007-1917-0

24. Y. P. Varshni, Comparative Study of Potential Energy Functions for Diatomic Molecules, Rev. Mod. Phys. 29 (1957) 664, https://doi.org/10.1103/RevModPhys.29.664

25. E. P. Inyang, E. P. Inyang, E. S. William, and E. E. Ibekwe, Study on the applicability of Varshni potential to predict the mass-spectra of the quark-antiquark systems in a nonrelativistic framework, arXiv:2101.00333.
26. C. O. Edet and P. O. Okoi, Any l-state solutions of the Schrödinger equation for $q$ - deformed Hulthén plus generalized inverse quadratic Yukawa potential in arbitrary dimensions, Rev. Mex. Fis. 65 (2019) 333, https://doi.org/ $10.31349 /$ RevMexFis.65.333

27. I. E. Okon, O. Popoola, and E. E. Ituen, Bound state solution to Schrödinger equation with Hulthén plus exponential Coulombic potential with centrifugal potential barrier using parametric Nikiforov-Uvarov method, Int. J. Recent Adv. Phys. 5 (2016) 1, https://doi.org/10.14810/ijrap.2016.5101.

28. O. Bayrak and I. Boztosun, Bound state solutions of the Hulthén potential by using the asymptotic iteration method, Phys. Scr. 76 (2007) 92, https://doi.org/10.1088/ 0031-8949/76/1/016

29. N. Tazimi and A. Ghasempour, Bound State Solutions of ThreeDimensional Klein- Gordon Equation for Two Model Potentials by NU Method, Adv. High Energy Phys. 2020 (2020) 2541837, https://doi.org/10.1155/2020/2541837

30. S. M. Ikhdair and J. Abu-Hasna, Quantization rule solution to the Hulthén potential in arbitrary dimension with a new approximate scheme for the centrifugal term, Phys. Scr. 83 (2011) 025002, https://doi.org/10.1088/ 0031-8949/83/02/025002

31. U. S. Okorie et al., Approximate solutions of the Schrödinger equation with energydependent screened Coulomb potential in D-dimensions, Eclét. Quím. J. 45 (2020), https://doi.org/10.26850/1678-4618eqj. v45.4.2020.p40-56

32. C. O. Edet, P.O. Okoi, Any l-state solutions of the Schrodinger equation for q-deformed Hulthén plus generalized inverse quadratic Yukawa potential in arbitrary dimensions, Rev Mex Fis, 65 (2019) 333. https: //doi.org/10.31349/ RevMexFis.65.333

33. C. O. Edet, U. S. Okorie, A. T. Ngiangia, and A. N. Ikot, Bound state solutions of the Schrödinger equation for the modified Kratzer potential plus screened Coulomb potential, Indian J. Phys. 94 (2020) 425, https://doi.org/10.1007/ s12648-019-01477-9

34. C. O. Edet, K. O. Okorie, H. Louis, and N. A. NzeataIbe, Any $l$-state solutions of the Schrödinger equation interacting with Hellmann-Kratzer potential model, Indian J. Phys. 94 (2020) 243, https://doi.org/10.1007/ s12648-019-01467-x

35. E. S. William, E. P. Inyang, and E. A. Thompson, Arbitrary $\ell-$ solutions of the Schrödinger equation interacting with HulthénHellmann potential model, Rev. Mex. Fis. 66 (2020) 730, https://doi.org/10.31349/RevMexFis.66.730

36. E. P. Inyang et al., Any $l$-state solutions of the Schrödinger equation interacting with class of Yukawa-Eckart potentials, Int. J. Innov. Sci. Eng. Technol. 7 (2020) 42.

37. C. O. Edet et al., Solutions of Schrödinger equation and thermal properties of generalized trigonometric Pöschl-Teller potential, Rev. Mex. Fis. 66 (2020) 824, https://doi.org/ 10.31349/RevMexFis.66.824

38. A. F. Nikiforov and V. B. Uvarov, Special Functions of Mathematical Physics (Birkhäuser, Basel, 1988), https://doi. org/10.1007/978-1-4757-1595-8 
39. E. P. Inyang et al., Approximate solutions of D-dimensional Klein-Gordon equation with Yukawa potential via NikiforovUvarov method, Indian J. Phys. (2021), https://doi. org/10.1007/s12648-020-01933-x

40. C. A. Onate and J. O. Ojonubah, Relativistic and nonrelativistic solutions of the generalized Pöschl-Teller and hyperbolical potentials with some thermodynamic properties, Int. J. Mod. Phys. E 24 (2015) 1550020, https : / / doi .org/10. $1142 / \mathrm{S} 0218301315500202$

41. O. Ebomwonyi, C. A. Onate, and O. E. Odeyemi, Application of formula method for bound state problems in Schrödinger equation, J. Appl. Sci. Environ. Manag. 23 (2019) 323, https://doi.org/10.4314/jasem.v23i2.19
42. W.-C. Qiang, Y. Gao, and R.-S. Zhou, Arbitrary l-state approximate solutions of the Hulthén potential through the exact quantization rule, Cent. Eur. J. Phys. 6 (2008) 356, https: //doi.org/10.2478/s11534-008-0041-1

43. O. Bayrak, G. Kocak, and I. Boztosun, Any l-state solutions of the Hulthén potential by the asymptotic iteration method, $J$. Phys. A 39 (2006) 11521, https: / / doi .org/10.1088/ $0305-4470 / 39 / 37 / 012$

44. S. M. Ikhdair, An improved approximation scheme for the centrifugal term and the Hulthén potential, Eur. Phys. J. A 39 (2009) 307, https://doi.org/10.1140/epja/ i2008-10715-2 